\title{
Variáveis que influenciam a produção de celulases e xilanase por espécies de Aspergillus
}

\author{
Marilia Ribeiro Sales ${ }^{(1)}$, Rosemery Batista de Moura(1), Tatiana Souza Porto(1), \\ Gorete Ribeiro de Macedo $^{(2)}$ e Ana Lúcia Figueiredo Porto(1)
}

\begin{abstract}
(1) Universidade Federal Rural de Pernambuco, Departamento de Morfologia e Fisiologia Animal, Rua Dom Manoel de Medeiros, s/no, Dois Irmãos, CEP 52171-900 Recife, PE. E-mail: mariliarsales@gmail.com, moura_mery@yahoo.com.br, portots@yahoo.com.br, analuporto@yahoo.com.br (2)Universidade Federal do Rio Grande do Norte, Departamento de Engenharia Química, Avenida Senador Salgado Filho, № 3.000, Campus Universitário, CEP 59.072-970 Natal, RN. E-mail: gomacedo@eq.ufrn.br
\end{abstract}

Resumo - O objetivo deste trabalho foi determinar as variáveis que influenciam a produção simultânea de celulases e xilanase por Aspergillus aculeatus URM 4953 e A. phoenicis URM 4924, com uso de bagaço de cana-de-açúcar como substrato. As variáveis avaliadas foram $\mathrm{pH}$ inicial, tempo de cultivo, concentração do substrato, agitação, concentração do inóculo, temperatura, tipo de bagaço de cana-de-açúcar e espécie de Aspergillus. Foi utilizado o planejamento fatorial fracionário $2^{8-4}$, com três repetições no ponto central. Todas as variáveis analisadas influenciaram a produção de pelo menos uma das enzimas. As maiores atividades enzimáticas observadas foram: celulases totais, $0,45 \mathrm{UI} \mathrm{mL}^{-1}$; endoglucanase, $0,60 \mathrm{UI} \mathrm{mL}^{-1}$; exoglucanase, $0,17 \mathrm{U} \mathrm{mL}^{-1}$; celobiase $6,42 \mathrm{UI} \mathrm{mL}^{-1}$; e xilanase, $30,05 \mathrm{U} \mathrm{mL}^{-1}$. As melhores condições para produção das enzimas foram: $\mathrm{pH}$ 6,0; tempo de cultivo de 168 horas; bagaço de cana-de-açúcar residual como substrato; e A. aculeatus URM 4953, que produziu as celulases e a xilanase simultaneamente. As variáveis que influenciam a produção simultânea das celulases e xilanase são pH, tempo de cultivo, tipo de bagaço de cana-de-açúcar e espécie de Aspergillus.

Termos para indexação: Aspergillus aculeatus, Aspergillus phoenicis, bagaço de cana-de-açúcar, celobiase, endoglucanase, exoglucanase.

\section{Variables that influence the production of cellulases and xylanase by Aspergillus species}

\begin{abstract}
The objective of this work was to determine the variables that influence the simultaneous production of cellulases and xylanase by Aspergillus aculeatus URM 4953 and A. phoenicis URM 4924, using sugarcane bagasse as substrate. Initial $\mathrm{pH}$, cultivation time, substrate concentration, agitation, inoculum concentration, temperature, sugarcane bagasse type and Aspergillus species were the variables evaluated. It was used the $2^{8-4}$ fractional factorial design, with three replicates in the central point. All analysed variables influenced at least one of the enzyme production. The highest enzymatic activities were: total cellulases, $0.45 \mathrm{UI} \mathrm{mL}^{-1}$; endoglucanase, $0.60 \mathrm{UI} \mathrm{mL}{ }^{-1}$; exoglucanase, $0.17 \mathrm{U} \mathrm{mL}^{-1}$; cellobiase, $6.42 \mathrm{UI} \mathrm{mL}^{-1}$; and xylanase, $30.05 \mathrm{U} \mathrm{mL}^{-1}$. The best conditions for enzyme production were: $\mathrm{pH}$ 6.0; cultivation time of 168 hours; residual sugarcane bagasse as substrate; and A. aculeatus URM 4953, which produced simultaneously all the cellulases and xylanase. The variables that influence the simultaneous production of cellulases and xylanase are $\mathrm{pH}$, cultivation time, sugarcane bagasse type, and Aspergillus species.
\end{abstract}

Index terms: Aspergillus aculeatus, Aspergillus phoenicis, sugarcane bagasse, cellobiase, endoglucanase, exoglucanase.

\section{Introdução}

O bagaço de cana-de-açúcar, um dos mais abundantes subprodutos derivados da indústria sucroalcooleira, é um resíduo fibroso do colmo, remanescente do processo de obtenção do caldo de cana para produção de açúcar e álcool. Ao ser hidrolisado, o produto apresenta uma fase líquida, constituída principalmente de glicose e xilose, entre outros açúcares (Bocchini et al., 2005).

$O$ bagaço de cana-de-açúcar tem sido utilizado como substrato para o cultivo de grande número de microrganismos, inclusive bactérias, leveduras e fungos filamentosos. Entretanto, esses últimos têm sido os mais utilizados, em razão da quantidade de enzimas e do enriquecimento proteico produzidos por eles. Temse comprovado que a bioconversão do bagaço pode ser economicamente vantajosa em casos como o da produção de enzimas (Pandey et al., 2000). O bagaço tem sido utilizado como substrato para produção de celulases e xilanase por várias espécies de Aspergillus, inclusive A. niger e A. phoenicis (Pandey et al., 2000; Sánchez, 2009). 
As celulases secretadas por fungos filamentosos são formadas por três componentes principais: as exoglucanases ou celobiohidrolases, as endoglucanases ou celodextrinases, e as celobiases ou $\beta$-glicosidases, essas últimas não são consideradas como celulases legítimas, mas desempenham um importante papel na hidrólise da celulose (Martins et al., 2008).

Para a hidrólise da celulose, a ação conjunta das enzimas endo-exo ocorre da seguinte forma: exoglucanases funcionam como exoenzimas que agem no final das cadeias e liberam celobiose como principal produto; endoglucanases agem de forma aleatória ao longo da cadeia e produzem novos sítios para ação das exoglucanases; e as $\beta$-glicosidases completam o processo por meio da hidrólise da celobiose e outros oligossacarídeos curtos a glicose (Martins et al., 2008). Também as celulases totais, conhecidas como enzimas do papel de filtro - FPase (Ghose, 1987) -, consistem de endoglucanases, exoglucanases e $\beta$-glicosidases (Zhang et al., 2006).

As xilanases possuem um importante papel na hidrólise do bagaço de cana por hidrolisar xilana, polissacarídeo formado por unidades monoméricas de xilose, que é um dos principais constituintes da hemicelulose (Heck et al., 2002; Dumitriu, 2005).

$\mathrm{O}$ uso do bagaço de cana-de-açúcar pode tornar viável e reduzir o custo da produção de celulases e xilanase. No entanto, as condições operacionais para a fermentação precisam ser otimizadas para a produção máxima na tecnologia industrial. $\mathrm{O}$ método clássico de otimização consiste em mudar uma determinada variável fixa e manter as demais fixas, o que é trabalhoso, consome tempo, especialmente quando se avalia um grande número de variáveis, e pode levar a resultados incertos e conclusões imprecisas. Essas limitações podem ser superadas com a utilização do planejamento fatorial, que possibilita a consideração dos efeitos dos fatores simultaneamente, o que otimiza a atividade da enzima e permite a realização de um número mínimo de experimentos (Heck et al., 2005; Alam et al., 2008). Assim, planejamentos fatoriais têm sido utilizados como técnicas confiáveis para o estudo da influência de múltiplos fatores na produção de enzimas (Silva et al., 2009).

O objetivo deste trabalho foi determinar variáveis que influenciama produção simultânea de celulases e xilanase por A. aculeatus URM 4953 e A. phoenicis URM 4924, com uso do bagaço de cana-de-açúcar como substrato, por meio de planejamento fatorial fracionário.

\section{Material e Métodos}

As linhagens de Aspergillus foram obtidas da Coleção de Culturas Micoteca URM (University Recife Mycology) da Universidade Federal de Pernambuco, Recife, PE: A. aculeatus URM 4953 e A. phoenicis URM 4924. As culturas foram mantidas em tubos que continham ágar extrato de malte esterilizado, incubados a $30^{\circ} \mathrm{C}$ por 30 dias.

$\mathrm{O}$ bagaço de cana-de-açúcar residual (BCR), procedente do processo industrial para obtenção do caldo de cana-de-açúcar, e o bagaço de cana-de-açúcar cru (BCC) foram cedidos pela Usina Ipojuca, Ipojuca, PE. A principal diferença entre o BCR e o BCC é que o primeiro é processado industrialmente, enquanto o BCC é obtido pelo método da compressão em uma moenda elétrica doméstica.

Ambos os tipos de bagaços, lavados com água potável e destilada, foram secos e moídos em processador de alimentos doméstico Walita Master Plus (Philips, Varginha, Brasil). O meio de cultivo utilizado para a produção de celulases e xilanase apresentou a seguinte composição: $0,2 \mathrm{~g} \mathrm{~L}^{-1}$ de $\mathrm{KH}_{2} \mathrm{PO}_{4} ; 0,13 \mathrm{~g} \mathrm{~L}^{-1}$ de $\left(\mathrm{NH}_{4}\right)_{2} \mathrm{SO}_{4} ; 0,03 \mathrm{~g} \mathrm{~L}^{-1}$ de $\mathrm{CO}\left(\mathrm{NH}_{2}\right)_{2} ; 0,03 \mathrm{~g} \mathrm{~L}^{-1}$ de $\mathrm{MgSO}_{4} \cdot 7 \mathrm{H}_{2} \mathrm{O} ; 0,03 \mathrm{~g} \mathrm{~L}^{-1}$ de $\mathrm{CaCl}_{2} ; 0,5 \mathrm{mg} \mathrm{L}^{-1}$ de $\mathrm{FeSO}_{4} \cdot 7 \mathrm{H}_{2} \mathrm{O} ; 0,156 \mathrm{mg} \mathrm{L}^{-1}$ de $\mathrm{MnSO}_{4} \cdot \mathrm{H}_{2} \mathrm{O} ; 0,14 \mathrm{mg} \mathrm{L}^{-1}$ de $\mathrm{ZnSO}_{4} \cdot 7 \mathrm{H}_{2} \mathrm{O} ; 0,14 \mathrm{mg} \mathrm{L}^{-1}$ de $\mathrm{CoCl}_{2}$ (Martins et al., 2008); e BCR ou BCC como substrato, nos níveis experimentais (Tabela 1).

$\mathrm{O}$ inóculo foi obtido de uma cultura em ágar e extrato de malte por sete dias, de ambas as espécies de Aspergillus. Os esporos foram suspensos em solução de Tween $80(0,01 \%)$ e inoculados em $50 \mathrm{~mL}$ do meio de cultivo contido em tubos de Erlenmeyer com capacidade para $250 \mathrm{~mL}$. A concentração final de

Tabela 1. Matriz estatística do planejamento fatorial dos níveis das variáveis experimentais para produção de celulases e xilanase por Aspergillus aculeatus URM $4953 \mathrm{e}$ A. phoenicis URM 4924.

\begin{tabular}{lccc}
\hline Variáveis & -1 & Ponto Central & +1 \\
\hline $\mathrm{pH}$ & 6 & 7,0 & 8 \\
Concentração de substrato $(\% \mathrm{p} / \mathrm{v})$ & 0,5 & 1,0 & 1,5 \\
Concentração do inóculo $\left(\right.$ esporos $\left.\mathrm{mL}^{-1}\right)$ & $10^{2}$ & $10^{3}$ & $10^{4}$ \\
Linhagem & A. phoenicis & Cultura mista & A. aculeatus \\
Substrato & $\mathrm{BCC}^{(1)}$ & Mistura $1: 1$ & $\mathrm{BCR}^{(2)}$ \\
Tempo de cultivo (horas) & 72 & 120 & 168 \\
Agitação (rpm) & 0 & 90 & 180 \\
Temperatura $\left({ }^{\circ} \mathrm{C}\right)$ & 30 & 35 & 40 \\
\hline
\end{tabular}

${ }^{(1)}$ Bagaço de cana-de-açúcar cru. ${ }^{(2)}$ Bagaço de cana-de-açúcar residual. 
esporos foi obtida de acordo com os três níveis desta variável $\left(10^{2}, 10^{3}\right.$ e $10^{4}$ esporos por $\mathrm{mL}$ ) (Tabela 1$)$. Os cultivos foram mantidos em agitador orbital, em diferentes rotações, temperatura e tempo de cultivo (Tabela 1) e, após esse período, foram centrifugados (5.000 g), e o sobrenadante obtido foi utilizado como extrato enzimático.

O extrato enzimático foi usado para determinar as atividades das celulases totais, endoglucanase, exoglucanase, celobiase e xilanase, bem como para dosar a quantidade de proteínas totais, com uso do kit para dosagem de proteínas micro BCA (Thermo Scientific, Rockford, EUA).

A atividade de celulases totais (FPase) foi determinada com uso do papel de filtro Whatmann $n^{0} 1$ (SigmaAldrich, Maidstone, Inglaterra) como substrato; a de endoglucanase foi determinada com uso de carboximetilcelulose - CMC (Sigma-Aldrich, St. Louis, EUA); e a atividade de celobiase foi avaliada com uso de kit comercial de glicose oxidase (Labtest, Lagoa Santa, Brasil), conforme Ghose (1987), com uso da celobiose (Sigma-Aldrich, Steinheim, Alemanha) como substrato. A atividade de exoglucanase foi determinada pelo método de Mawadza et al., (2000) modificado, tendo-se incubado $250 \mu \mathrm{L}$ do extrato enzimático com $500 \mu \mathrm{L}$ de solução $1 \%(\mathrm{p} / \mathrm{v})$ de celulose microcristalina Sigmacell type 50 (Sigma-Aldrich, St. Louis, EUA), em solução tampão citrato (50 $\left.\mathrm{mmol} \mathrm{L}^{-1}, \mathrm{pH} 5,0\right)$ a $50^{\circ} \mathrm{C}$ durante 2 horas. A atividade xilanolítica foi determinada de acordo com Bailey et al. (1992), com uso de xilana de endoderma de aveia (Sigma-Aldrich, Steinheim, Alemanha) como substrato. Em razão de o meio de cultivo conter açúcares redutores que poderiam interferir no resultado das atividades enzimáticas, o controle foi realizado com adição do reativo DNS para inativação da enzima (Miller, 1959), antes da incubação desta com seu respectivo substrato.

Para todas as atividades enzimáticas, a unidade determinada foi a quantidade de enzima necessária para liberar $1 \mu \mathrm{mol}$ de glicose ou xilose por minuto de reação, conforme a enzima avaliada. As atividades enzimáticas foram expressas em atividade volumétrica $\left(\mathrm{U} \mathrm{mL}^{-1}\right)$ e atividade relativa (percentagem da atividade volumétrica do ensaio em que a enzima apresentou maior atividade), para comparação dos resultados entre as enzimas estudadas.
A fim de obter maiores níveis de produção de celulases e xilanase por A. aculeatus URM 4953 e A. phoenicis URM 4924, foi necessário estudar e variar consideravelmente as condições de cultivo. A seleção das variáveis foi realizada com base em dados já descritos na literatura (Bocchini et al., 2002; Alam et al., 2008; Martins et al., 2008). Foram estudados níveis de oito variáveis na produção de celulases: $\mathrm{pH}$, tempo de cultivo, concentração do substrato $(\%$ p/v), agitação (rpm), concentração do inóculo, temperatura, tipo do bagaço de cana-de-açúcar e linhagens de Aspergillus isoladas e em cultura mista (Tabela 1).

No módulo de análise e planejamento de experimentos do programa Statistica 8.0 (Statsoft INC, 2008), foi obtido um planejamento fatorial fracionário $2^{8-4}$, que resultou em 16 ensaios diferentes entre si e três repetições no ponto central (ensaios 17 a 19), o que totalizou 19 ensaios por meio da distribuição dos níveis das variáveis experimentais (Tabela 2). A atividade relativa das enzimas celulases totais, exoglucanase, endoglucanase, celobiase e xilanase foi obtida como variáveis resposta e analisadas no programa Statistica 8.0 (Statsoft INC, 2008), no módulo de análise dos componentes principais. A determinação dos efeitos das variáveis e a significância dos coeficientes de regressão foram determinadas pelo teste $\mathrm{t}$ de Student.

\section{Resultados e Discussão}

A atividade de celulases totais (L) não foi detectada no ensaio 10 (Tabela 2). As mais altas atividades de celulases totais $\left(0,45\right.$ e $\left.0,27 \mathrm{UI} \mathrm{mL}^{-1}\right)$ foram obtidas no menor $\mathrm{pH}(6,0)$ e no maior tempo de cultivo (168 horas) com o BCR, como substrato para a produção da enzima, e A. aculeatus URM 4953. Todas as variáveis foram significativas $(\mathrm{p}<0,05)$, exceto a concentração de substrato, porém as variáveis $\mathrm{pH}\left(\mathrm{X}_{1}\right)$, linhagem $\left(\mathrm{X}_{4}\right)$, tipo de bagaço $\left(\mathrm{X}_{5}\right)$ e tempo de cultivo $\left(\mathrm{X}_{6}\right)$ apresentaram os maiores efeitos (Tabela 3).

Silva et al. (2009) observaram efeito significativo da concentração de substrato sobre a produção de celulases totais por Aspergillus phoenicis, com uso de resíduo de uva como substrato. Esses autores avaliaram as concentrações de substrato $0,5,1,0 \mathrm{e}$ $1,5 \% \mathrm{p} / \mathrm{v}$, e observaram atividade máxima de $0,11 \mathrm{UI}$ $\mathrm{mL}^{-1}$, na concentração $1 \%$ de resíduo. No entanto, no presente trabalho, a concentração de substrato foi a única variável que não apresentou efeito significativo 
sobre a produção de celulases totais por A. phoenicis URM 4924 e A. aculeatus URM 4952, com bagaço de cana-de-açúcar como substrato. No presente trabalho, a linhagem A. phoenicis URM 4924 teve cerca de quatro vezes mais atividade que a de $A$. phoenicis descrita por Silva et al. (2009), no maior nível de produção da enzima.

Alam et al. (2008) realizaram otimização do processo de produção de celulase por Trichoderma harzianum, por meio da bioconversão do estado líquido de lodo de esgoto doméstico, e encontraram atividade máxima de $6,9 \mathrm{UI} \mathrm{mL}^{-1}$ a $32,5^{\circ} \mathrm{C}$, concentração de substrato a $0,75 \%$, pH inicial 5,0, inóculo 2,0\% e $175 \mathrm{rpm}$. A temperatura, o pH inicial e o inóculo tiveram efeitos significativos, enquanto a concentração de substrato não, o que está de acordo com os resultados encontrados no presente trabalho.

Quanto à endoglucanase $(\mathrm{N})$, a atividade variou de

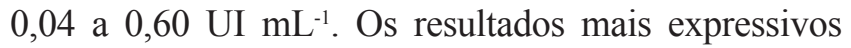
de atividade desta enzima foram nos maiores níveis de agitação (180 rpm) e tempo de cultivo (168 horas) e no menor nível de temperatura $\left(30^{\circ} \mathrm{C}\right)$ (Tabela 2$)$. Pode-se sugerir que, além dos níveis das variáveis citados anteriormente, deve ser utilizado o BCR como substrato para a produção de endoglucanase por A. aculeatus URM 4953 em pH 6,0, concentração do substrato de $1,5 \%$ p/v e concentração do inóculo de $10^{4}$.

Silva et al. (2009) observaram efeito positivo significativo da concentração de resíduo de uva $(p<0,05)$ a $1,5 \% \quad(p / v)$. Resultado similar foi encontrado no presente trabalho, uma vez que os níveis de concentração de substrato estudados $(0,5,1,0$ e $1,5 \%$ ) produziram efeito positivo sobre a produção de endoglucanase.

Quanto à produção de exoglucanase (E), a linhagem A. aculeatus URM 4953 foi a mais promissora para produção dessa enzima, com uso do BCR, e as variáveis significativas foram: $\mathrm{pH}$, concentração de substrato e temperatura de cultivo (Tabela 3). Essas duas últimas tiveram efeitos positivos, ou seja, quanto maior a concentração de substrato e a temperatura de cultivo, maior foi a atividade enzimática. $\mathrm{O} \mathrm{pH}$, no entanto, teve efeito negativo. A mais alta atividade de exoglucanase foi de $0,17 \mathrm{U} \mathrm{mL}^{-1}$ no $\mathrm{pH} 6,0$, concentração de substrato $1,5 \%$ e temperatura de cultivo a $40^{\circ} \mathrm{C}$.

Tabela 2. Distribuição dos níveis das variáveis experimentais, para produção de enzimas do complexo celulolítico e da xilanase, com as respostas da atividade relativa (\%) das enzimas após cultivo de Aspergillus aculeatus URM 4953 e A. phoenicis URM 4924, com uso de bagaço de cana-de-açúcar cru (BCC) e residual (BCR) como substratos.

\begin{tabular}{|c|c|c|c|c|c|c|c|c|c|c|c|c|c|c|}
\hline \multirow[t]{2}{*}{ Ensaio } & \multicolumn{8}{|c|}{ Fatores experimentais ${ }^{(1)}$} & \multicolumn{5}{|c|}{ Atividade relativa das enzimas $(\%)^{(2)}$} & \multirow[t]{2}{*}{ Proteínas totais $\left(\mu \mathrm{g} \mathrm{mL}^{-1}\right)$} \\
\hline & $\mathrm{X}_{1}$ & $\mathrm{X}_{2}$ & $\mathrm{X}_{3}$ & $\mathrm{X}_{4}$ & $\mathrm{X}_{5}$ & $\mathrm{X}_{6}$ & $\mathrm{X}_{7}$ & $\mathrm{X}_{8}$ & $\mathrm{~L}$ & $\mathrm{E}$ & $\mathrm{N}$ & $\mathrm{B}$ & $\mathrm{X}$ & \\
\hline 1 & 6 & 0,5 & $10^{2}$ & A. phoenicis & $\mathrm{BCC}$ & 72 & 0 & 30 & 9 & 13 & 13 & 100 & 8 & 16,91 \\
\hline 2 & 8 & 0,5 & $10^{2}$ & A. aculeatus & $\mathrm{BCR}$ & 72 & 180 & 40 & 10 & 14 & 13 & 3 & 9 & 20,27 \\
\hline 3 & 6 & 1,5 & $10^{2}$ & A. aculeatus & $\mathrm{BCR}$ & 168 & 0 & 40 & 62 & 100 & 20 & 20 & 66 & 10,51 \\
\hline 4 & 8 & 1,5 & $10^{2}$ & A. phoenicis & $\mathrm{BCC}$ & 168 & 180 & 30 & 16 & 19 & 97 & 2 & 82 & 13,28 \\
\hline 5 & 6 & 0,5 & $10^{4}$ & A. aculeatus & BCR & 168 & 180 & 30 & 100 & 18 & 100 & 6 & 52 & 17,70 \\
\hline 6 & 8 & 0,5 & $10^{4}$ & A. phoenicis & $\mathrm{BCC}$ & 168 & 0 & 40 & 5 & 9 & 7 & 1 & 6 & 14,48 \\
\hline 7 & 6 & 1,5 & $10^{4}$ & A. phoenicis & $\mathrm{BCC}$ & 72 & 180 & 40 & 9 & 16 & 41 & 76 & 94 & 15,58 \\
\hline 8 & 8 & 1,5 & $10^{4}$ & A. aculeatus & BCR & 72 & 0 & 30 & 9 & 16 & 14 & 1 & 13 & 11,46 \\
\hline 9 & 6 & 0,5 & $10^{2}$ & A. aculeatus & $\mathrm{BCC}$ & 168 & 180 & 40 & 10 & 16 & 14 & 22 & 48 & 13,52 \\
\hline 10 & 8 & 0,5 & $10^{2}$ & A. phoenicis & BCR & 168 & 0 & 30 & 0 & 0 & 13 & 3 & 0 & 18,20 \\
\hline 11 & 6 & 1,5 & $10^{2}$ & A. phoenicis & $\mathrm{BCR}$ & 72 & 180 & 30 & 26 & 26 & 32 & 3 & 31 & 10,67 \\
\hline 12 & 8 & 1,5 & $10^{2}$ & A. aculeatus & $\mathrm{BCC}$ & 72 & 0 & 40 & 8 & 7 & 9 & 1 & 6 & 19,14 \\
\hline 13 & 6 & 0,5 & $10^{4}$ & A. phoenicis & BCR & 72 & 0 & 40 & 6 & 15 & 15 & 10 & 12 & 10,69 \\
\hline 14 & 8 & 0,5 & $10^{4}$ & A. aculeatus & $\mathrm{BCC}$ & 72 & 180 & 30 & 25 & 37 & 43 & 4 & 100 & 20,15 \\
\hline 15 & 6 & 1,5 & $10^{4}$ & A. aculeatus & $\mathrm{BCC}$ & 168 & 0 & 30 & 10 & 0 & 57 & 3 & 8 & 13,97 \\
\hline 16 & 8 & 1,5 & $10^{4}$ & A. phoenicis & $\mathrm{BCR}$ & 168 & 180 & 40 & 13 & 19 & 13 & 1 & 7 & 17,55 \\
\hline 17 & 7 & 1,0 & $10^{3}$ & Cultura mista & Mistura 1:1 & 120 & 90 & 35 & 7 & 7 & 14 & 4 & 10 & 15,84 \\
\hline 18 & 7 & 1,0 & $10^{3}$ & Cultura mista & Mistura 1:1 & 120 & 90 & 35 & 5 & 13 & 10 & 10 & 11 & 13,91 \\
\hline 19 & 7 & 1,0 & $10^{3}$ & Cultura mista & Mistura 1:1 & 120 & 90 & 35 & 8 & 14 & 13 & 8 & 10 & 13,94 \\
\hline
\end{tabular}


A atividade de celobiase (B) variou entre 0,05 e 6,42 UI $\mathrm{mL}^{-1}$. Os resultados mais elevados de atividade de celobiase foram obtidos no menor nível de $\mathrm{pH}(6,0)$ e tempo de cultivo (72 horas), com BCC como substrato para a produção da enzima por A. phoenicis URM 4924 (Tabela 2). As variáveis $\mathrm{pH}$, linhagem, tipo de bagaço e tempo de cultivo foram significativas quanto à atividade de celobiase (Tabela 3).

A maior atividade de xilanase $(\mathrm{X})$ foi de $30,05 \mathrm{U} \mathrm{mL}^{-1}$, e os maiores valores de atividade dessa enzima ocorreram no maior nível da variável agitação (180 rpm), com BCC como substrato (Tabela 2). O tipo de substrato foi a única variável que teve efeito significativo (Tabela 3).

Bocchini etal. (2002) relataram que o tempo de cultivo e a interação entre o tempo de cultivo e a concentração de xilana como substrato tiveram efeito significativo na produção de xilanase por Bacillus circulans D1; os autores obtiveram atividade que variou de 3,99 a 19,39 U mL $\mathrm{mL}^{-1}$. Heck et al. (2006) concluíram que a mudança do $\mathrm{pH}$ e da temperatura podem melhorar a produção de xilanase consideravelmente. No presente trabalho, não se observou efeito das variáveis concentração de substrato, $\mathrm{pH}$, temperatura e tempo de cultivo sobre a atividade de xilanase (Tabela 2).

Diversas linhagens e espécies de microrganismos têm sido estudadas para a produção de celulases em diferentes fontes de carbono. Trichoderma reesei, uma das espécies mais estudadas para produção de enzimas celulolíticas, foi importante para maior produção de celobiase ( $\beta$-glicosidase). No entanto, essa espécie não é superior a $A$. phoenicis na produção de $\beta$-glicosidase, o que justifica o uso de culturas mistas para a produção mais eficiente de celulases (Silva et al., 2009).

No presente trabalho, também foi investigada a produção de celulases e xilanase por cultura mista de
A. phoenicis URM 4924 e A. aculeatus URM 4953; no entanto, os ensaios com cultura mista (ensaios 17 a 19, pontos centrais) tiveram cerca de dez vezes menos atividade que os melhores ensaios estudados para as culturas isoladas. Logo, o cultivo misto de A. phoenicis URM 4924 e A. aculeatus URM 4953 não é recomendado para a produção de todas as celulases e xilanase em índices elevados.

Para a hidrólise da celulose a monômeros de carboidrato, é necessária uma ação conjunta entre todas as enzimas do complexo celulolítico (Mawadza et al., 2000), o que leva a investigar, entre os ensaios com uma única linhagem, aquele em que foram produzidas maiores quantidades de celulases simultaneamente.

A componente principal 1 (PC1) foi responsável pela explicação de $46,80 \%$ da variação dos resultados, e a PC2 representou 22,38\%, o que correspondeu no plano cartesiano a $69,18 \%$ da variação dos resultados totais. Na PC1, a combinação de celulases e xilanase teve peso negativo. $\mathrm{Na} \mathrm{PC} 2$, os resultados foram contrastantes: celulases totais (L) e endoglucanase (N) com peso positivo, e xilanase (X), exoglucanase (E) e celobiase (B) com peso negativo. Isto significa que as celulases e a xilanase tendem a resultados de peso negativo, especialmente porque a $\mathrm{PC} 1$ apresenta as enzimas com resultados negativos e explica $46,80 \%$ dos resultados (Figura 1).

Na projeção plana multivariada dos ensaios (escore dos ensaios) (Figura 2), é possível observar que o ensaio 3 está posicionado no quadrante inferior esquerdo (peso negativo para PC1 e PC2) e o 5 no quadrante superior direito (peso negativo para $\mathrm{PC} 1$ e positivo para $\mathrm{PC} 2$ ), e são os mais distantes do eixo de intersecção do PC1 com a PC2, o que indica que se destacaram na produção enzimática, pois estão localizados na mesma posição das variáveis na projeção plana multivariada das

Tabela 3. Efeitos das variáveis na produção de celulases e xilanase, celulase total (L), exoglucanase (E), endoglucanase (N), celobiase (B) e xilanase (X).

\begin{tabular}{|c|c|c|c|c|c|}
\hline Variáveis & $\mathrm{L}\left(\mathrm{UI} \mathrm{mL} \mathrm{mL}^{-1}\right)$ & $\mathrm{E}\left(\mathrm{U} \mathrm{mL}^{-1}\right)$ & $\mathrm{N}\left(\mathrm{UI} \mathrm{mL} \mathrm{m}^{-1}\right)$ & $\mathrm{B}\left(\mathrm{UI} \mathrm{mL} \mathrm{mL}^{-1}\right)$ & $\mathrm{X}\left(\mathrm{U} \mathrm{mL}^{-1}\right)$ \\
\hline$\overline{\mathrm{pH}}$ & $-18,25^{*}$ & $-10,38 *$ & $-10,38^{*}$ & $-28,00 *$ & $-12,00$ \\
\hline Concentração de substrato $(\% \mathrm{p} / \mathrm{v})$ & $-1,50$ & $10,13 *$ & $8,13 *$ & $-5,25$ & 9,00 \\
\hline Concentração do inóculo & $4,50 *$ & $-8,13$ & $9,88^{*}$ & $-6,50$ & 5,25 \\
\hline Linhagem & $18,75^{*}$ & $11,38 *$ & $4,88^{*}$ & $-17,00^{*}$ & 7,75 \\
\hline Tipo do bagaço & $16,75^{*}$ & $11,38 *$ & $-7,62 *$ & $-20,25 *$ & $-20,25$ \\
\hline Tempo de cultivo & $14,25 *$ & 4,63 & $17,62 *$ & $-17,50^{*}$ & $-0,50$ \\
\hline Agitação (rpm) & $12,50 *$ & 0,63 & $25,63 *$ & $-2,75$ & $38,00 *$ \\
\hline Temperatura do cultivo $\left({ }^{\circ} \mathrm{C}\right)$ & $-9,00 *$ & $8,38 *$ & $-29,63 *$ & 1,50 & $-5,75$ \\
\hline Média & $17,79 *$ & $18,89 *$ & $28,32 *$ & $14,63 *$ & $30,16^{*}$ \\
\hline
\end{tabular}

*Significativo a $5 \%$ de probabilidade, pelo teste T de Student. 
variáveis (carregamento das variáveis) (Figura 1). Ao se correlacionar os dados dos escores e carregamento, observa-se também que o ensaio 5 teve maiores resultados para celulases totais (L) e endoglucanase (N) do que o ensaio 3 que, por sua, vez teve maiores

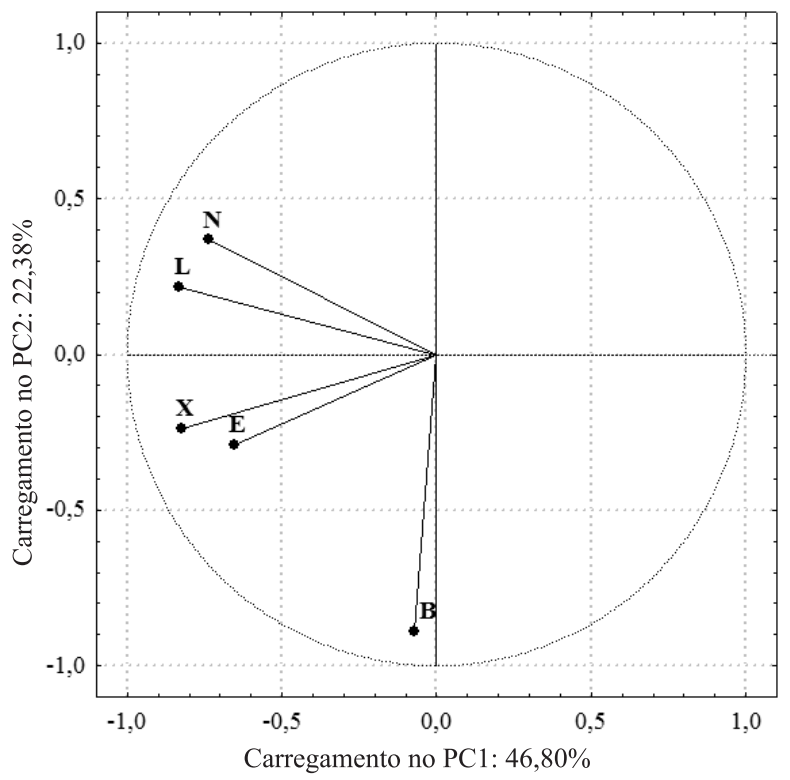

Figura 1. Projeção plana multivariada das variáveis, quanto à atividade relativa $(\%)$ de celulases totais $(\mathrm{L})$, exoglucanase $(\mathrm{E})$, endoglucanase $(\mathrm{N})$, celobiase $(\mathrm{B})$ e xilanase $(\mathrm{X})$. Carregamento das variáveis dos primeiro e segundo componentes principais. O plano formado por estes componentes reproduz $69,18 \%$ da informação original.

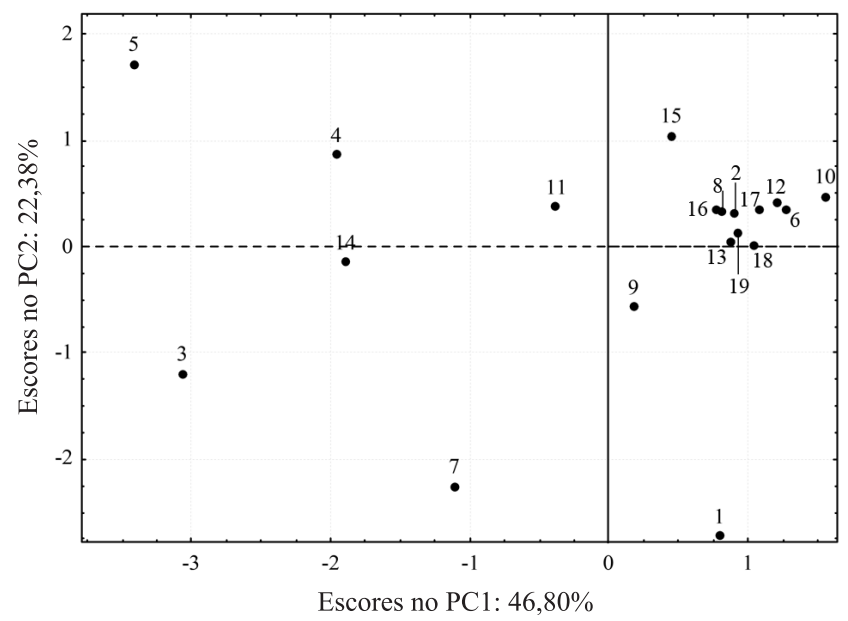

Figura 2. Projeção plana multivariada dos ensaios, para a atividade relativa de celulases totais, exoglucanase, endoglucanase, celobiase e xilanase. O plano formado por estes componentes reproduz $69,18 \%$ da informação original. resultados para xilanase (X) e exoglucanase (E) do que o ensaio 5, o que pode ser comprovado pela análise da atividade relativa das enzimas (Tabela 2 ).

Assim, os resultados obtidos no planejamento estatístico fracionário mostraram que, entre os ensaios realizados, os que apresentaram atividades mais elevadas das celulases e da xilanase, simultaneamente, foram os ensaios 3 e 5 , que tiveram como similaridade: $\mathrm{pH}$ 6,0, tempo de cultivo de 168 horas, BCR como substrato para produção das enzimas, e a linhagem A. aculeatus URM 4953.

É provável que o BCR tenha sido melhor indutor na produção de enzimas em razão de ter sido submetido a processos de aquecimento, moagem e recompressão industriais, enquanto que na primeira etapa de obtenção do BCC, o colmo passa por uma moeda elétrica, onde é apenas comprimido. O processo para obtenção de caldo de cana-de-açúcar, industrialmente, consiste na extração do caldo primário e secundário, em que o bagaço é moído e recomprimido para extração de quase todo o açúcar solúvel, para um melhor aproveitamento do processo. Assim, com uso do BCC, os fungos filamentosos tiveram disponível uma quantidade inicial de açúcar no meio de cultivo, o que pode ter causado efeito repressivo na síntese enzimática (Botella et al., 2007).

\section{Conclusões}

1. As variáveis $\mathrm{pH}$, tempo de cultivo, tipo de bagaço de cana-de-açúcar e espécie de Aspergillus influenciam a produção simultânea de celulases e xilanase.

2. A espécie $A$. aculeatus URM 4953 produz celulases e xilanase simultaneamente, o que favorece a hidrólise da celulose, com uso de bagaço de cana-de-açúcar residual como substrato.

\section{Agradecimentos}

À Coordenação de Aperfeiçoamento de Pessoal de Nível Superior e ao Conselho Nacional de Desenvolvimento Científico e Tecnológico, pelo apoio financeiro; à Usina Ipojuca S/A, por prover o bagaço de cana-de-açúcar usado na pesquisa; ao Prof. Dr. Benício Barros Neto, pelo auxílio nas análises estatísticas; e à Coleção de Culturas Micoteca URM da Universidade Federal de Pernambuco, por ceder as linhagens fúngicas utilizadas neste trabalho. 


\section{Referências}

ALAM, M.Z.; MUYIBI, S.A.; WAHID, R. Statistical optimization of process conditions for cellulase production by liquid state bioconversion of domestic wastewater sludge. Bioresource Technology, v.99, p.4709-4716, 2008.

BAILEY, M.J.; BIELY, P.; POUTANEN, K. Interlaboratory testing of methods for assay of xylanase activity. Journal of Biotechnology, v.23, p.257-270, 1992.

BOCCHINI, D.A.; ALVES-PRADO, H.F.; BAIDA, L.C.; ROBERTO, I.C.; GOMES, E.; DA SILVA, R. Optimization of xylanase production by Bacillus circulans D1 in submerged fermentation using response surface methodology. Process Biochemistry, v.38, p.727-731, 2002.

BOCCHINI, D.A.; OLIVEIRA, O.M.M.F.; GOMES, E.; SILVA, R.D. Use of sugarcane bagasse and grass hydrolysates as carbon sources for xylanase production by Bacillus circulans D1 in submerged fermentation. Process Biochemistry, v.40, p.3653-3659, 2005.

BOTELLA, C.; DIAZ, A.; ORY, I. de; WEBB, C.; BLANDINO, A. Xylanase and pectinase production by Aspergillus awamori on grape pomace in solid state fermentation. Process Biochemistry, v.42, p.98-101, 2007.

DUMITRIU, S. Polysaccharides: structural diversity and functional versatility. $2^{\text {nd }}$ ed. New York: Marcel Dekker, 2005. 1204p.

GHOSE, T.K. Measurement of cellulase activities. Pure and Applied Chemistry, v.59, p.257-268, 1987.

HECK, J.X.; FLÔRES, S.H.; HERTZ, P.F.; AYUB, M.A.Z. Optimization of cellulase-free xylanase activity produced by Bacillus coagulans BL69 in solid-state cultivation. Process Biochemistry, v.40, p.107-112, 2005.
HECK, J.X.; FLÔRES, S.H.; HERTZ, P.F.; AYUB, M.A.Z. Statistical optimization of thermo-tolerant xylanase activity from Amazon isolated Bacillus circulans on solid-state cultivation. Bioresource Technology, v.97, p.1902-1906, 2006.

HECK, J.X.; HERTZ, P.F.; AYUB, M.A.Z. Cellulase and xylanase production by isolated Amazon Bacillus strains using soybean industrial residue based solid-state cultivation. Brazilian Journal of Microbiology, v.33, p.213-218, 2002.

MARTINS, L.F.; KOLLING, D.; CAMASSOLA, M.; DILLON, A.J.P.; RAMOS, L.P. Comparison of Penicillium echinulatum and Trichoderma reesei cellulases in relation to their activity against various cellulosic substrates. Bioresource Technology, v.99, p.1417-1424, 2008.

MAWADZA, C.; HATTI-KAUL, R.; ZVAUYA, R.; MATTIASSON, B. Purification and characterization of cellulases produced by two Bacillus strains. Journal of Biotechnology, v.83, p.177-187, 2000.

MILLER, G.L. Use of dinitrosalicylic acid reagent for determination of reducing sugar. Analitical Chemistry, v.31, p.426-428, 1959.

PANDEY, A.; SOCCOL, C.R.; NIGAM, P.; SOCCOL, V.T. Biotechnological potential of agro-industrial residues. I: Sugarcane bagasse. Bioresource Technology, v.74, p.69-80, 2000.

SÁNCHEZ, C. Lignocellulosic residues: biodegradation and bioconversion by fungi. Biotechnology Advances, v.27, p.185-194, 2009.

SILVA, L.D.; LOPES, F.C.; SILVEIRA, S.T.; BRANDELLI, A. Production of cellulolytic enzymes by Aspergillus phoenicis in grape waste using response surface methodology. Applied Biochemistry and Biotechnology, v.152, p.295-305, 2009.

ZHANG, Y.H.P.; HIMMEL, M.E.; MIELENZ, J.R. Outlook for cellulase improvement: screening and selection strategies. Biotechnology Advances, v.24, p.452-481, 2006.

Recebido em 11 de julho de 2010 e aprovado em 18 de outubro de 2010 